\title{
The impact of sleep deprivation in military surgical teams: a systematic review
}

\author{
Rachael SV Parker, ${ }^{1}$ P Parker $^{2}$
}

${ }^{1}$ Emergency Medicine Department, Royal Preston Hospital, Preston, UK ${ }^{2}$ Department of Trauma \& Orthopaedics, University Hospitals, Birmingham NHS Foundation Trust, Birmingham, UK

\section{Correspondence to}

Col Paul Parker, Department of Trauma \& Orthopaedics, University Hospitals, Birmingham NHS Foundation Trust, Birmingham B15 2TH, UK; Paul.Parker@uhb.nhs.uk, parker_paul@hotmail.com

Received 27 March 2016 Revised 28 July 2016 Accepted 7 August 2016 Published Online First 13 September 2016

\section{CrossMark}

To cite: Parker RSV, Parker P. J R Army Med Corps

2017;163:158-163.

\begin{abstract}
Background Fatigue in military operations leads to safety and operational problems due to a decrease in alertness and performance. The primary method of counteracting the effects of sleep deprivation is to increase nightly sleep time, which in operational situations is not always feasible. History has taught us that surgeons and surgical teams are finite resources that cannot operate on patients indefinitely.
\end{abstract}

Methods A systematic review was conducted using the search terms 'sleep' and 'deprivation' examining the impact of sleep deprivation on cognitive performance in military surgical teams. Studies examining outcomes on intensive care patients and subjects with comorbidities were not addressed in this review.

Results Sleep deprivation in any 'out-of-hours' surgery has a significant impact on overall morbidity and mortality. Sleep deprivation in surgeons and surgical trainees negatively impacts cognitive performance and puts their own and patients' health at risk. All published research lacks consensus when defining 'sleep deprivation' and 'rested' states. It is recognised that it would be unethical to conduct a well-designed randomised controlled trial, to determine the effects of fatigue on performance in surgery; however, there is a paucity between surrogate markers and applying simulated results to actual clinical performance. This requires further research. Recommended methods of combating fatigue include: prophylactically 'sleep-banking' prior to known periods of sleep deprivation, napping, use of stimulant or alerting substances such as modafinil, coordinated work schedules to reduce circadian desynchronisation and regular breaks with enforced rest periods.

Conclusions A forward surgical team will become combat-ineffective after 48 hours of continuous operations. This systematic review recommends implementing on-call periods of no more than 12 hours in duration, with adequate rest periods every 24 hours. Drug therapies and sleep banking may, in the short term, prevent negative effects of acute sleep deprivation.

\section{INTRODUCTION}

Fatigue in military operations leads to safety and operational problems due to a decrease in alertness and performance. ${ }^{1}$ The primary method of counteracting the effects of sleep deprivation is to increase nightly sleep time but in operational situations this is not always feasible. Recovery of cognitive function is accomplished more rapidly after acute total sleep deprivation than chronic partial sleep deprivation-a state more commonly encountered in everyday life; the American Association of Sleep Medicine suggests prophylactically 'banking' sleep before a known period of deprivation.

\section{Key messages}

- A forward surgical team cannot operate on patients indefinitely, and will likely become combat-ineffective after 48 hours of continuous operating.

- Sleep deprivation results in decreased cognitive performance, alertness and increased attentional failures and errors.

- Drug therapies like modafinil and sleep banking can be effective short-term agents for preventing the negative effects of sleep deprivation.

- Understanding and applying sleep physiology to military working conditions is essential to improve the functionality of the deployed surgical team and ultimately patient outcomes.

Besides rapid eye movement (REM) and non-REM (NREM) types of sleep, the body can induce a state of recovery sleep that is not equal to normal sleep. $^{2} 3$

Recovery sleep has a shorter sleep latency, a shorter time spent in stage one of NREM sleep, increased sleep efficiency and greater amounts of slow-wave and REM sleep. Just one night of recovery sleep can reverse the effects of acute total sleep deprivation; conversely, three 8-hour periods of sleep are not enough to restore cognitive performance from chronic sleep deprivation. ${ }^{2}$ This paper examines the physiology of sleep, sleep deprivation and how this may apply to the deployed small surgical team.

\section{THE HISTORICAL MILITARY PROBLEM}

At the height of conflict in Afghanistan in 2010 (Operation Herrick), the large UK/US R3 Hospital in Camp Bastion had five surgical teams allowing two or three teams at a time to have 'days-off' away from the operating theatre, although other clinical work was continued. The small R2 hospital in Sierra Leone in 2015 had one orthopaedic surgeon and one general surgeon, inevitably resulting in extended periods, if not continuous, on-call durations, which was the same staffing level as Camp Bastion on Herrick IV in 2006. Although not expected to have high numbers or a concentrated inflow of patients, it is a resource-limited team. As the UK military transitions back to such contingency operations, the question arises as to how many casualties can such a team and its resources manage before becoming exhausted. 
This question of surgeons operating in the face of fatigue is not a new one, as witnessed by the six month operation of forward surgical teams during the Kokoda-Buna campaign in 1942-3. ${ }^{4}$ Part of the Pacific War of World War II, the campaign consisted of a series of battles fought between Japanese and Allied-primarily Australian forces-in what was then the Australian territory of Papua. After the fall of Singapore, the Australian government feared that Japan would invade mainland Australiaan attack that Australia was ill-prepared to counter. The Kokoda track itself runs $100 \mathrm{~km}$ across coastal lowlands into the Solomon Sea; isolated, rugged and passable only by foot, this area gives rise to hot humid days, intensely cold nights, torrential rainfall and endemic tropical diseases. ${ }^{5}$ These campaigns have entered Australian mythology because of the bravery exhibited against the enormous odds in an inhospitable terrain. ${ }^{4}$ The chain of evacuation used during these campaigns is similar to the current model implemented by the Defence Medical Services (DMS). Early on in the campaigns a surgeon, Captain DR Leslie, 2/9th Australian General Hospital, attached to 2/6th Field Ambulance was requested to move forward with the advance to assist with early management of casualties, with very little equipment as his orders were: "Do not do any operating - the conditions will not allow it. Your job is to see that the wounded are travelling light". ${ }^{4}$

Conditions at the time were truly testing (Figure 1$)^{6}$ and the evacuation of many patients was delayed by days due to heavy rain. Inevitably, among close quarters and challenging environment to exercise aseptic technique, infection was rife. It was initially impossible to decide what hours the single surgical team could work, without rest, on the steady influx of injured servicemen. It was clear, however, that no rest in 24 hours would render the service wholly depleted and that it would cease after 2 or, at most, 3 days.

A decision was therefore made to operate for 18-hour periods only, and then by default, men requiring surgery were deliberately left as there was no alternative; this allowed the team to continue to function for several months. In December of the campaign, two surgeons arrived at Soputa and worked in two 12-hour shifts, which enabled every wounded man to be

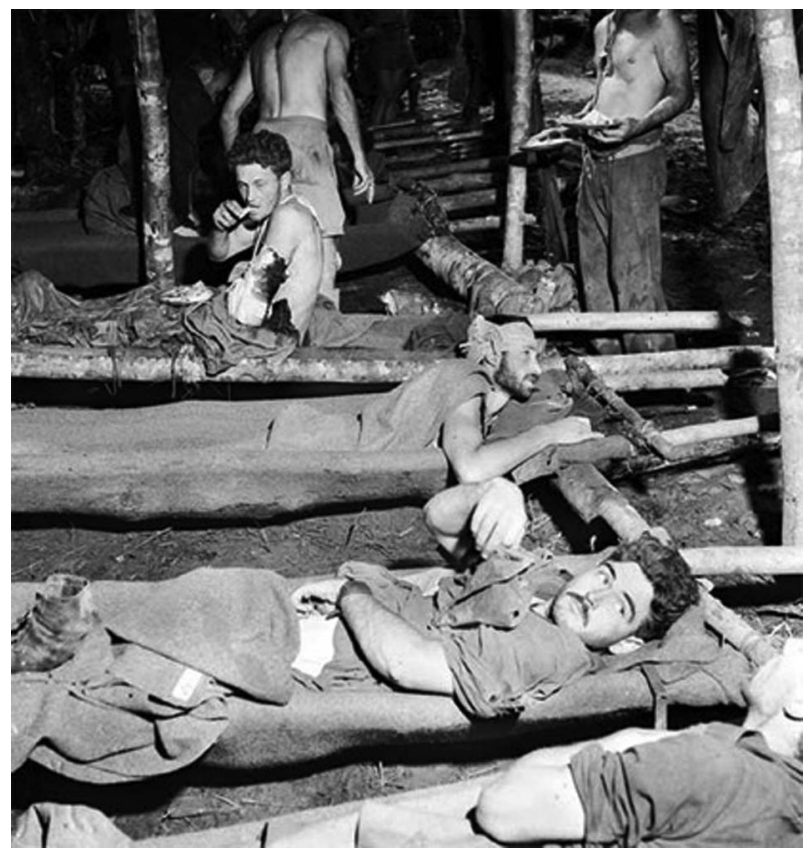

Figure 1 'Australian infantry, wounded while attacking Oivi, are treated afterwards at an advanced dressing station. November 1942.' The Kokoda Trust. operated on and for 3 days, operation theatre work lasted 24 hours a day. With the clear advantage of preserving life and maintaining service functionality, the model of two surgeons operating in shifts was continued and expanded to include four teams in total: two surgical teams operating by day and two teams operating by night. ${ }^{4}$ With our more advanced knowledge of sleep and sleep manipulation, how can we expect a modern two-surgeon team to perform in 2016?

\section{METHODS}

A systematic search was conducted of EMBASE, PubMed, Cochrane Library and the Journal of the Royal Army Medical Corps archive from inception to 2016, restricted to English-language articles to identify all studies examining the impact of sleep deprivation on cognitive performance in military surgical teams in PubMed. ${ }^{7}$ The search strategy was:

1. Sleep $(\mathrm{MeSH})$ AND Deprivation $(\mathrm{MeSH})$ gave 10,556 results

2. English-language articles only, 9600 results

3. Systematic reviews only, 147 results

Types of studies and participants included were: randomised controlled trials; systematic reviews and non-randomised comparative studies, examining the effect of sleep deprivation on military and civilian surgical teams, surgeons and surgical trainees. Comparisons were made between work patterns of aviation and healthcare systems; and between interventions including prophylactic sleep and activity breaks; stimulants and hypnotics; strategic scheduling of working hours and improved working conditions. Outcomes include the direct impact of sleep deprivation on surgeons and healthcare workers; the effects of sleep deprivation on cognitive performance as applied to surgical outcomes and the effect of shift work on cognitive function. Fourteen studies met the criteria and underwent qualitative and quantitative data synthesis (Figure 2). Intensive care patients and studies examining subjects with comorbidities were not addressed in this review. Outcomes relating to neuroimaging analyses, pain and mental health were not addressed in this review.

\section{RESULTS}

\section{Physiology of sleep}

Sleep is defined as a 'naturally recurring state, in an absence or decrease in consciousness with relatively suspended sensory activity, and inactivity of nearly all voluntary muscles'. It is a more easily reversible state than that of hibernation or being in a coma. ${ }^{8}$ As the earth rotates around its own axis and orbits the sun, a 24-hour cycle of light and dark is created, leaving all organisms on the planet with an intrinsic clock, an endogenous oscillation of approximately 24 hours duration known as the circadian rhythm. ${ }^{9}$ The circadian rhythm dictates the ideal time for restorative sleep cycles. Our pressure to sleep is greatest between 01:00 and 04:00 and there is a second, smaller increase in sleepiness 12 hours later between 13:00 and 16:00. This 'afternoon low-point' is created by an innate homeostatic sleep propensity and is not related to eating a large or calorific lunchtime meal.

High-levels of adenosine secreted at appropriate points during the circadian rhythm induce sleepiness by inhibiting bodily processes associated with wakefulness. Adequate sleep produces no daytime dysfunction or sleepiness. 9 . The terms sleepiness and fatigue are often used interchangeably, but they are distinct phenomena. Sleepiness denotes a tendency to fall asleep, fatigue refers to an overwhelming sense of tiredness and feeling of exhaustion, associated with impaired physical and/or 


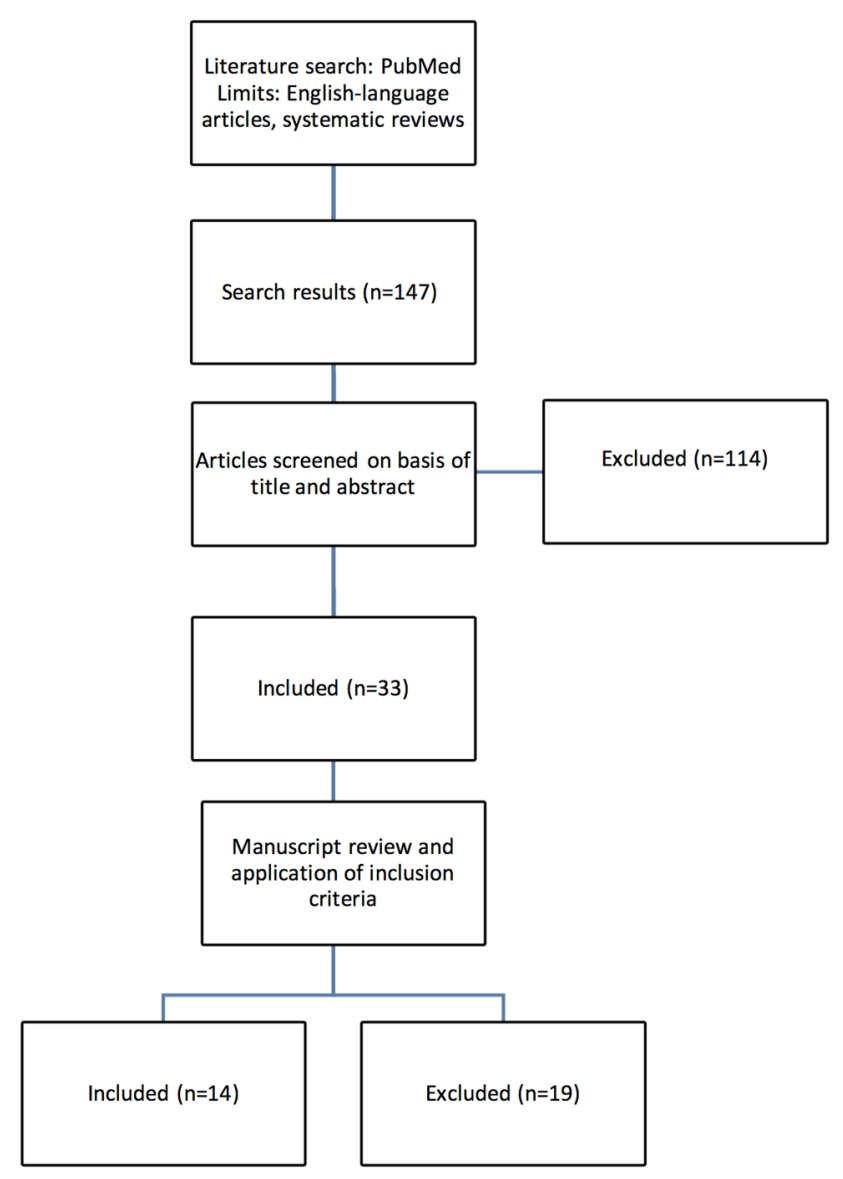

Figure 2 Summary of the literature search used in PubMed and exclusion process.

cognitive deprivation; both often coexist as a consequence of sleep deprivation. ${ }^{10}$ Sleep can be inefficient and inadequate when taken at the wrong time of day. There are several stages to sleep, standardised in 1968-it comprises cycles of NREM subclassified into stages N1-N3 and REM sleep. ${ }^{11}$ Theories on the functions of sleep are multiple and still require much in the way of scientific research to develop a strong evidence base.

\section{Restoration}

Adequate sleep improves wound healing as sleep deprivation impairs the immune system and can reduce white blood cell levels by $20 \%$; this challenge to the immune system alters sleep behaviour and thus a cycle of deterioration is created. Rats kept awake indefinitely develop skin lesions, hyperphagia and hypothermia and eventually, all subjects die of sepsis. ${ }^{12}$ Insomnia is the difficulty of falling and staying asleep. A permanent complete absence of sleep, whether due to psychological stress, an inconsistent sleep schedule or willed behaviour is impossible.

A rare condition called familial fatal insomnia exists-a hereditary prion autosomal dominant disease, characterised by the progressive loss of the ability to sleep, progressive sympathetic hyperactivity-increased perspiration and salivation, constipation, impotence, tachycardia, systemic hypertension and mild fever and attenuation of autonomic and hormonal oscillations. ${ }^{13}$ Patients appear apathetic and unable to pay sustained attention to their surroundings; in more advanced stages, the vigilance level declines to a persistent stupor and a vegetative state may precede death. Disease presents in the sixth decade of life and the clinical course is usually $<2$ years from onset to death. ${ }^{14}$
Regardless of this medical phenomenon, the end result of permanent insomnia is death.

\section{Ontogenesis}

Neonates average 18 hours of REM sleep a day to maximise brain development; deprivation can lead to behavioural problems, permanent sleep disruption, a decrease in brain mass and an increase in neuronal cell death. Many mammals sleep for a larger proportion of each 24 -hour period when very young. ${ }^{15}$

\section{Memory processing}

There is a correlation between sleep and complex memory function; 'working memory' keeps information active to allow it to be further processed and support higher cognitive functions such as decision-making, reasoning and episodic memory. ${ }^{16}$ Sleep is an essential part of memory and learning-emotion boosts consolidation of events in the declarative memory system and REM sleep fosters this consolidation. ${ }^{3}{ }^{17}$ It is clear that the sleeping brain provides an ideal environment for solidifying newly learned information in the brain. ${ }^{18}$ In sleep-deprived subjects who slept for 26 min over 4 days, working memory suffered $38 \%$ impairment. ${ }^{16}$

\section{Sleep deprivation}

Sleep deprivation has two main forms: acute total sleep deprivation where an individual has no sleep in 24 hours and chronic partial sleep deprivation: at least 1 week of consecutive 24-hour periods with 6 or fewer hours sleep. Lack of consensus on the definitions of these terms places limitations on research in this field due to inconsistencies in study designs, accuracy and reproducibility of evidence found. Peets ${ }^{19}$ defines chronic partial sleep deprivation as 'less than normal but not zero hours sleep', whereas other studies only declare 'rested' and 'unrested' states, which leaves ambiguity in quantifying quality and quantity of sleep obtained by subjects. ${ }^{20}$

Most people, regardless of sex or race prefer 7-8 hours of sleep a night and each day with insufficient sleep forms a sleep debt. Problems manifested by a sleep debt occur at predictable times of day according to the circadian rhythm and are most noticeable during the second 12-hour cycle, with the 3 hour nadir between 13:00 and 16:00. This sleep debt creates lapses in attention, a decrease in short-term memory capacity and impairs decision-making skills. Apart from the sleep debt causing detriment to a military personnel's ability to perform cognitive tasks, one must also be aware of the negative impact of sleep inertia, a 5-30 min period of lethargy or sluggishness immediately after waking, an essential period during which performing important military tasks should be avoided. ${ }^{21}$

Larger sleep debt leads to mental, physical and emotional fatigue. When the sleep debt is great enough the brain experiences microsleeps: 10-60 s episodes where the brain enters an NREM sleep-like state, similar to blackouts, irrespective of what a person is doing. ${ }^{22}$ The individual does not know this momentary blackout has occurred. Apart from diminished abilities to perform higher-cognitive function, frontal regions in the brain are extremely sensitive to homeostatic sleep pressure. There is no quantifiable measure of how much sleep debt one can accumulate or an average sleep debt that is applicable between individuals. ${ }^{23}$ Microsleeps in conjunction with attentional lapses, and an individual variation in sleep debt propensity can have significant impacts on people working together as a team-particularly in a military surgical environment. 
Counteracting the effects of sleep deprivation

Prophylactic sleep and activity breaks

Despite the short periods of sleep inertia immediately following naps, napping and increasing the length of night-time sleep in advance of a known period of prolonged wakefulness, or sleep 'banking', have been recognised to be successful methods in ameliorating and preventing the effects of acute sleep deprivation, respectively. Both study subjects and researchers agree that the benefits associated with these naps far outweigh the potential risks. $^{24}{ }^{25}$ When commencing shifts that run through the night or through the circadian trough, nothing is lost from reporting for work in the afternoon of a 17-hour night shift instead of the morning of a 24-hour shift. In this way, surgeons avoid becoming cognitively tired during the daytime when they are expected to continue working the evening night as well. ${ }^{9}$

One recommendation to ameliorate the physical impact of fatigue on night shifts, particularly in surgeons performing operations more than 2 hours in duration, is to incorporate breaks of $20 \mathrm{~s}$ every $20 \mathrm{~min}$, throughout the procedure. During the $20 \mathrm{~s}$ break surgeons stretch their arms, necks and shoulders. This significantly improves muscle strength, precision, fatigue and surgeon well-being while reducing intraoperative events, and the scheduled breaks do not prolong the operation. ${ }^{9}$

\section{Stimulants and hypnotics}

Stimulant medications maintain alertness and performance in sleep-deprived military fighter aircrew during combat operations without significant postflight symptoms of racing heart, drugged feeling, nausea, dizziness and headache. Both surgery and military aviation have similar working environments-an unpredictability of sortie initiation or the arrival of casualties, thus requiring surgical teams and pilots alike to remain vigilant during long periods of relative inactivity, while waiting for short periods of task-saturated mission-critical activity. ${ }^{1}$

Other stimulants commonly used include caffeine and nicotine. Caffeine is an adenosine antagonist-slowing the action of hormones in the brain that normally cause somnolence, therefore maintaining alertness. ${ }^{24}$ Nicotine decreases an individual's sleeping time, which by definition increases hours of wakefulness, but it does decrease daytime drowsiness. ${ }^{2}$ Alcohol has several mechanisms of action: following consumption, alcohol initially acts as a sedative inducing somnolence but there is a rebound effect several hours later as alcohol disrupts sleep and decreases the total amount of REM sleep, hence many alcoholics also experience insomnia. ${ }^{2}$ However, these countermeasures do nothing to address the actual source of fatigueinsufficient sleep. ${ }^{24}$

Gore et $\mathrm{al}^{1}$ report that during 111 combat flight sorties averaging 7.6 hours in duration, stimulants were used during $35 \%$, an average 2.8 hours after take off. There is a statistically significant use of in-flight stimulants during the circadian trough, longer sortie duration and preflight hypnotic use. Regulations allow pilots to consume either 5 or $10 \mathrm{mg}$ dextroamphetamine every 4 hours in flight, or $200 \mathrm{mg}$ modafinil every 8 hours. All aircrew were authorised a single dose of hypnotic medication (temazepam 15 or $30 \mathrm{mg}$, zolpidem $10 \mathrm{mg}$, zaleplon $10 \mathrm{mg}$ ) for preflight sleep at least 12,6 or 4 hours prior to takeoff, respectively. ${ }^{1}$ This complies with the American Academy of Sleep Medicine (AASM) recommendation of increasing sleep before embarking on a known period of sleep deprivation, ${ }^{26}$ although pilots employing this method of preflight rest were more than twice as likely to use stimulants in flight. ${ }^{1}$
The term neuroenhancement refers to improvement in the cognitive, emotional and motivational functions of healthy individuals through, among other things, the use of drugs. ${ }^{27}$ Modafinil is an alerting substance, also used to treat excessive sleepiness due to narcolepsy, obstructive sleep apnoea and shift work sleep disorder. ${ }^{9}$ It is preferable to amphetamine and dextroamphetamine as it is less addictive and has fewer of amphetamine's side effects, ${ }^{9} 28$ which include insomnia, anxiety, increased alertness and decreased hunger. ${ }^{29}$ The exact mechanism of modafinil is unclear but it is thought to increase the concentration of monoamines and hypothalamic histamines, thus promoting a state of wakefulness. It has proven useful in increasing certain aspects of cognitive function in sleep-deprived physicians after night shifts. Areas of cognition improved were memory, attention, flexible thinking and decision making, but there was no effect on psychomotor performance. Despite these positive findings, after taking the stimulant modafinil, surgeons reported trouble sleeping on call, if given the opportunity, and at home post-on call. ${ }^{9} 27$

However, repeated doses of modafinil are unable to prevent deterioration over a longer period of sleep deprivation despite maintaining wakefulness, and may even induce overconfidence in a person's own cognitive performance. ${ }^{27}$ Therefore, the use of modafinil as a medium for doctors to take on extended work hours in a 'safe' manner is irresponsible and without regard for the health and safety of the doctors themselves, and the safety of patients in the long term. ${ }^{9}$

Passive fatigue measures are already in place to maximise alertness and performance in the form of duty hour limitations. In the UK, doctors work 48 hours per week (the general European Union maximum), Scandinavia sees physicians work $37-48$ hours in average over 4 weeks and the USA places restrictions at 80 work hours a week. ${ }^{30}$ The UK and Scandinavia have a maximum of 16 hours work in a 24-hour period. ${ }^{9}{ }^{31}$ These working regulations apply to civilian and military medical personnel. Other passive measures include improved sleeping conditions for deployed service personnel, napping and strategic scheduling; however, certain extreme situations dictate that these implementations are not feasible. Although study findings indicate stimulant use was effective and well tolerated, operational fatigue countermeasures in aviation and healthcare alike should focus on minimising circadian desynchronisation and preflight (or preoperative periods) hypnotic use by promoting consistent scheduling, as seen in the Kokoda-Buna conflict with alternating 12-hour shifts between surgical teams. ${ }^{14}$

Applying normal sleep physiology to a military task force recognises the effect of circadian rhythm on operating within the morning nadir, between 01:00 and 04:00 twilight hours-a surgical team must operate at this time to ensure continuous patient care in hostile conditions, but operational planners must take into account the negative effects of sleep inertia and physiology when conducting operations in austere environments.

\section{Fatigue in surgery and anaesthesia}

Long hours are almost considered a tradition during medical training. ${ }^{20}$ Sleep deprivation has been shown to increase attention failures in intensive care unit doctors and medical errors in internal medicine doctors, there is an increased risk of percutaneous injuries in junior doctor's first year of training and an increase in motor accidents in doctors driving home after shifts. ${ }^{9}$ Albergo et $a l^{32}$ analysed cognitive performance of orthopaedic surgical trainees before and after 24 hours of on-call duty. Cognitive tests were performed after sleeping for at least 6 hours and after being on call-sleeping for $<3$ hours. This 
study concluded that sleep deprivation after 24 hours on-call duty affects cognitive performance, increasing the number of errors and omissions.

Eleven studies examined by Sturm et $a l,{ }^{20}$ assessed performance using simulation-based methods when a participant was rested and/or fatigued. Three studies reported performance when fatigued as either improved or stayed the same. Two studies showed an increase in errors because of sleep deprivation. Despite these initial findings, none of the papers using simulated performances applied the effects of the results to clinical outcomes. Another study found that for operations performed on a normal working day, complications were $45 \%$ more likely to occur when the doctor had been on call the day before. $^{20}$

Healthcare professionals who work longer than 12 consecutive hours, or work when they have not obtained sufficient sleep, are putting their patients' health at risk, damaging their own health and if they drive home when they are drowsy, also put the health of the general public at risk. ${ }^{10}$ Among anaesthetic trainees, $63 \%$ reported their ability to perform professionally as 'low' after a night shift and a concerning 15\% admitted to having a fatigue-related motor accident during his or her training programme. ${ }^{33}$ Procedures performed by cardiothoracic surgeons 'out-of-hours' between 21:00 and 05:00 and those ending between 23:00 and 07:30, resulted in a significantly higher rate of postoperative sepsis in patients. ${ }^{34}$ Asfour et al ${ }^{34}$ concluded that sleep deprivation in out-of-hours non-cardiothoracic surgery, including trauma and general surgery had a significant impact on overall morbidity and mortality.

There are limitations to the evidence on fatigue and surgical performance and one commonly encountered problem is a lack of consensus on defining key terms such as 'rested' and 'unrested'. 'Rested' has been defined as approximately 5-6 hours of sleep, however, other research states this length of sleep is still inadequate to qualify as being 'rested'. ${ }^{29}$ furthermore, the impact of chronic sleep deprivation was not included. The literature is not always clear in determining if medical professionals are surgical trainees or surgeons and the difference in level of training means that the majority of the evidence is based on trainees (any doctor with between 1 and 9 or more years' experience), ${ }^{35}$ thus questioning the ability to extrapolate conclusions to fully trained surgeons. Finally, more data are needed to know if less experienced surgical trainees or newly qualified surgeons are more likely to make errors when sleep deprived than senior surgeons. ${ }^{20}$

Simulated performance is of questionable value; however, in recognition that it would be unethical to conduct a welldesigned randomised controlled trial to determine the effects of fatigue on performance in surgery, simulation has become an accepted surrogate. Simulation is not always realistic and the relationship between surrogate markers and actual clinical performance requires further study. ${ }^{20}$ When clinical performance was measured, there was no inclusion of procedures cancelled due to surgeon absenteeism, surgeons feeling too tired or sleepdeprived surgeons altering their workload to take into account their level of fatigue to only perform standard uncomplicated procedures.

Two studies gave subjects pre-assessment proficiency training in a simulated environment; therefore, a learning effect may have biased the results. Over the first three night shifts and with an increasing effect of fatigue, performance times for the simulated tasks initially improved, and then plateaued at baseline levels for the remaining two nights. ${ }^{9}$ This evidence suggests that surgeons can adapt and prepare for a known task even when sleep-deprived. ${ }^{9}{ }^{20}$ With this in mind, simulation and repeatedly practicing tasks or competencies prior to operational deployment could increase the functionality of a deployed small surgical team, by using the learning effect to increase the baseline performance level of individuals and the team as whole.

Whether a subject is acutely sleep deprived or chronically sleep deprived has unique effects on different timescales, although the neurobehavioural impact of each state may be similar once sleep deprivation is established. There are greater detriments in performance seen in junior doctors than senior physicians or consultants, suggesting that healthcare professionals who are more skilled, experienced and knowledgeable are less likely to show decline in career-related tasks when sleep deprived. ${ }^{9}$ Irrespective of this, fatigue is seen to affect the cognitive domains of attention, decision-making and retrieving longterm memory regardless of one's experience or skill. Equally, it is important to acknowledge that there are many factors contributing to an individual's performance in any task beyond the remits of number of hours slept or which point in one's circadian rhythm an assessment is performed. ${ }^{20} 33$

Limiting the length of a shift has the benefits of reducing the impact of acute and chronic sleep deprivation, although it does not provide relief from sleep inertia or the effects of working against one's circadian rhythm. The primary rationale behind implementation of work-hour restrictions takes into account the single most effective way of combating sleep deprivation-sleeping more. The aviation industry limits pilots who fly for more than 8 hours to have at least 16 hours of rest before being reassigned; this has been practiced for more than 75 years. ${ }^{19}$ Surgeons work with the same intensity and responsibility as pilots. International healthcare systems have been slow to follow a protocol of this nature.

With regard to doctors-in-training, there is the question of balancing the risk of fatigue-related errors with continuity-related errors. Fundamentally, the mind cannot function if the body and brain are deprived of sleep, but a reduction in working hours is not a panacea for patient safety. Work-hour restrictions are in place to reduce the severity of sleep deprivations, prevent very serious consequences of sleep-deprived cognitive impairment and ultimately produce healthy, caring doctors who deliver optimal care. ${ }^{19}$ A morally challenging concept in austere situations, the realisation that material and cognitive surgical resources are finite was unforgivingly executed in the Kokoda-Buna conflict. To preserve the energy and functionality of the surgical team, a long-term view had to be taken in order for the surgical team to exist beyond the casualties sustained in the first battle, the price for this was some loss of life during the mandatory rest periods. ${ }^{4}$ Learning from lessons of the past we should now be able to put in place contingency plans to ensure, where possible, continuous resources are available to operate on incoming casualties and still preserve the well-being and lifespan of the forward surgical team.

\section{CONCLUSION}

The lessons of 1942 hold true today. A modern UK forward surgical team will likely become combat-ineffective after 48 hours of continuous operating and after this time, patients will die. Improving our understanding of sleep physiology, as applied to the deployed forward surgical team (FST), is paramount to the functionality of personnel and outcomes for patients alike. Drug therapies and sleep banking may, in the short term, prevent the negative effects of acute sleep deprivation, and simple interventions like short pauses at regular intervals can help to improve precision and fatigue. On-call periods must be limited to no 
more than 12 hours in duration, with adequate rest periods in 24 hours, before the next working shift commences.

However, the current evidence base is weak, due to a lack of consensus among current literature with regard to definitions of sleep deprivation, the ethical inability to carry out true randomised controlled trials beyond simulated environments, a deficit in clinical application of surrogate markers for cognitive impairment due to sleep deprivation and non-committal conclusions about the impact of sleep deprivation as a single factor impacting on surgical outcomes. Absence of evidence is not an absence of effect; in the crucible of conflict, it is owed to service personnel, surgical or otherwise, to optimise life-saving and limbsaving ability.

Contributors PP contributed to conception of research aims. RSVP contributed to the systematic review of the literature, interpreted the data and drafted all sections of the article. PP provided critical intellectual comment to revise the drafts. All authors approved the version of the article submitted for consideration.

Competing interests None declared.

Provenance and peer review Not commissioned; externally peer reviewed.

\section{REFERENCES}

1 Gore R, Webb T, Hermes E. Fatigue and stimulant use in military fighter aircrew during combat operations. Aviat Space Environ Med 2010;81:719-27.

2 Alhola P, Polo-Kantola P. Sleep deprivation: impact on cognitive performance. Neuropsychiatr Dis Treat 2007;3:553-67.

3 Wiesner CD, Pulst J, Krause F, et al. The effects of selective REM-sleep deprivation on the consolidation and affective evaluation of emotional memories. Neurobiol Learn Mem 2015;122:131-41.

4 Grogan R. The operation of forward surgical teams in the Kokoda-Buna campaigns. ANZ J Surg 1998;68:68-73

5 McCarthy D. Chapter 2: the Island barrier. In: McCarthy D, ed. Volume V-South-West Pacific area - first year: Kokoda to Wau. Canberra: Australian War Memorial, 1962:34-83.

6 The Kokoda Trust. Australian Infantry, wounded while attacking Oivi, are treated afterwards at an advanced dressing station. November 1942. http://kokoda. commemoration.gov.au/casualties/kokoda-milne-bay-buna-gona-casualty-lists.php (accessed Mar 2015).

7 Hemingway $\mathrm{P}$, Bereton N. What is a systematic review? http://www.whatisseries.co. uk (accessed Apr 2009). http://www.medicine.ox.ac.uk/bandolier/painres/download/ whatis/syst-review.pdf

8 Oxford Dictionaries. Definition of sleep in English. http://www.oxforddictionaries. com/definition/english/sleep (accessed Jul 2016).

9 Amirian I. The impact of sleep deprivation on surgeons' performance during night shifts. Dan Med J 2014;61:B4912.

10 Rogers $\mathrm{A}$. The effects of fatigue and sleepiness on nurse performance and patient safety. In: Hughes R, ed. Patient safety and quality: an evidence-based handbook for nurses. Rockville: Agency for Healthcare Research and Quality, 2008:1036-60.

11 Silber M, Ancoli-Israel S, Bonnet $\mathrm{M}$, et al. The visual scoring of sleep in adults. J Clin Sleep Med 2007:3:121-31.

12 Zager A, Anderson ML, Ruiz FS, et al. Effects of acute and chronic sleep loss on immune modulation of rats. Am J Physiol Regul Integr Comp Physiol 2007;293: R504-509.
13 Montagna $\mathrm{P}$, Gambetti $\mathrm{P}$, Cortelli $\mathrm{P}$, et al. Familial and sporadic fatal insomnia. Lancet Neurol 2003:2:167-76.

14 Cortelli P, Gambetti P, Montagna P, et al. Fatal familial insomnia: clinical features and molecular genetics. J Sleep Res 1999;8(Suppl 1):23-9.

15 Mirmiran M, Scholtens J, van de Poll N, et al. Effects of experimental suppression of active (REM) sleep during early development upon adult brain and behaviour in the rat. Brain Res 1983;283:277-86.

16 Stickgold R. Sleep-dependent memory consolidation. Nature 2005;437:1272-8.

17 Tempesta D, De Gennaro L, Natale V, et al. Emotional memory processing is influenced by sleep quality. Sleep Med 2015;16:862-70.

18 Alger S, Chambers A, Cunningham T, et al. The role of sleep in human declarative memory consolidation. Curr Top Behav Neurosci 2015;25:269-306.

19 Peets A, Ayas NT. Restricting resident work hours: the good, the bad, and the ugly. Crit Care Med 2012:40:960-6.

20 Sturm L, Dawson D, Vaughan R, et al. Effects of fatigue on surgeon performance and surgical outcomes: a systematic review. ANZ J Surg 2011;81:502-9.

21 Giam G. Effects of sleep deprivation with reference to military operations. Ann Acad Med Singap 1997:26:88-93.

22 Koslowsky M, Babkoff $\mathrm{H}$. Meta-analysis of the relationship between total sleep deprivation and performance. Chronobiol Int 1992;9:132-6.

23 Van Dongen H, Maislin G, Mullington J, et al. The cumulative cost of additional wakefulness: dose-response effects on neurobehavioural functions and sleep physiology from chronic sleep restriction and total sleep deprivation. Sleep 2003;26:117-26

24 Hartzler BM. Fatigue on the flight deck: the consequences of sleep loss and the benefits of napping. Accid Anal Prev 2014;62:309-18.

25 Ruggiero JS, Redeker NS. Effects of napping on sleepiness and sleep-related performance deficits in night-shift workers: a systematic review. Biol Res Nurs 2014;16:134-42.

26 Watson NF, Morgenthaler T, Chervin R, et al., American Academy of Sleep Medicine Board of Directors. Confronting drowsy driving: the American Academy of Sleep Medicine Perspective. J Clin Sleep Med 2015;11:1335-6.

27 Repantis D, Schlattmann P, Laisney 0 , et al. Modafanil and methylphenidate for neuroenhancement in healthy individuals: a systematic review. Pharmacol Res 2010;62:187-206.

28 Pigeau R, Naitoh P, Buguet A, et al. Modafinil, d-amphetamine and placebo during 64 hours of sustained mental work. I. Effects on mood, fatigue, cognitive performance and body temperature. J Sleep Res 1995:4:212-28.

29 Taheri S, Lin L, Austin D, et al. Short sleep duration is associated with reduced leptin, elevated ghrelin, and increased body mass index. PLoS Med 2004;1:e62.

30 Fletcher K, Davis S, Underwood W, et al. Systematic review: effects of resident work hours on patient safety. Ann Intern Med 2004;141:851-7.

31 BMA. European working time directive. BMA. http://bma.org.uk/support-at-work/ ewtd (accessed Oct 2015).

32 Albergo Jl, Fernández M, Zaifrani L, et al. How does sleep deprivation during 24 hours on call duty affect the cognitive performance of orthopaedic residents? Rev Esp Cir Ortop Traumatol 2016;60:113-18.

33 Pikovsky 0, Oron M, Shiyovich A. The impact of sleep deprivation on sleepiness, risk factors and professional performance in medical residents. Isr Med Assoc $J$ 2013;15:739-44

34 Asfour L, Asfour V, McCormack D, et al. In surgeons performing cardiothoracic surgery is sleep deprivation significant in its impact on morbidity or mortality? Interact Cardiovasc Thorac Surg 2014;19:479-89.

35 GMC. Medical career structure. GMC. http://www.gmc-uk.org/Medical_career_ structure__doctors_in_training.pdf_25417075.pdf (accessed Oct 2015). 